\title{
Atividade acaricida in vitro de Glechon spathulata Benth. sobre teleóginas de Rhipicephalus (Boophilus) microplus
}

\author{
Acaricide activity in vitro of Glechon spathulata Benth. On engorged females of Rhipcephalus \\ (Boophilus) microplus
}

\begin{abstract}
Andréia Buzatti ${ }^{\mathrm{I}}$ Felipe da Silva Krawczak ${ }^{\mathrm{I}}$ Felipe Lamberti Pivoto ${ }^{\mathrm{I}}$ Fernanda Silveira Flores Vogel ${ }^{\mathrm{I}}$ Sônia de Avila Botton ${ }^{\mathrm{I}}$ Gilberto Dolejal ZanettiI ${ }^{\mathrm{II}}$ Melânia Palermo Manfron $^{\mathrm{II}}$ Luís Antonio Sangioni ${ }^{*}$
\end{abstract}

RESUMO

O objetivo deste estudo foi avaliar a atividade acaricida in vitro de extratos vegetais de Glechon spathulata (manjeroninha do campo) sobre teleóginas de Rhipicephalus (Boophilus) microplus. Fêmeas ingurgitadas desse carrapato foram coletadas de bovinos mestiços de uma propriedade do município de São Pedro do Sul, Rio Grande do Sul, Brasil. A experimentação in vitro consistiu na realização de testes de imersão de fêmeas ingurgitadas. O extrato bruto seco e a fração clorofórmica utilizados nos testes in vitro foram preparados na concentração de $2 \%$ e diluídos em água e etanol, sendo todos os tratamentos realizados em triplicata. $O$ extrato bruto seco de G. spathulata retomado em etanol a $70 \%$ apresentou uma eficácia média de $99 \%$, enquanto que quando o mesmo extrato foi diluído em água, demonstrou eficácia média de $72 \%$. Observou-se que no controle etanol a $70 \%$ houve diferença na eclodibilidade dos ovos, porém não interferiu no peso da postura. A fração clorofórmica de G. spathulata não apresentou eficácia no ensaio efetuado. Os resultados obtidos nesse estudo demonstraram que o extrato hidroetanólico a $70 \%$ de $\boldsymbol{G}$. spathulata apresenta eficácia acaricida in vitro. Este estudo é o primeiro relato de ação acaricida de G. spathulata.

Palavras-chave: carrapato, G. spathulata, fitoterápico, plantas medicinais, controle parasitário.

\section{ABSTRACT}

The aim of this study was to evaluate the in vitro acaricide efficiency of Glechon spathulata extracts on engorged females of Rhipcephalus (Boophilus) microplus. Engorged ticks were collected from crossbred cattle from one farm in São Pedro do Sul municipality, Rio Grande do Sul, Brazil. In vitro experiments consisted on the immersion tests of engorged females in solutions of $\boldsymbol{G}$. spathulata, where treatments were made at triplicate with fractions and extracts at $2 \%$ concentration, diluted in water and ethanol. Crude dry extract of G. spathulata suspended in $70 \%$ ethanol presented an efficiency average of $99 \%$, while the same extract diluted in water demonstrated efficiency average of $72 \%$. It was observed in $70 \%$ ethanol control difference in egg eclodibility; however there was no interference in egg weight. The chloroform fraction of $\boldsymbol{G}$. spathulata showed no efficacy in the test. The results obtained suggest that the hydroethanolic extract at $70 \%$ of $\boldsymbol{G}$. spathulata has significant acaricide activity in vitro. This research is the first communication of acaricidal effect of. $\boldsymbol{G}$. spathulata.

Key words: tick, G. spathulata, phytotherapy, medicinal plants, parasitic control.

\section{INTRODUÇÃO}

Rhipicephalus (Boophilus) microplus se destaca como um dos ectoparasitas que mais prejudica o desempenho produtivo dos bovinos, em consequência das ações espoliadora, mecânica e tóxica (FRAGA et al., 2003). O uso dos acaricidas constitui o principal instrumento de controle desse carrapato em bovinos (FARIAS, 1999). Entretanto, a utilização dos carrapaticidas resultou no desenvolvimento de populações de ixodideos resistentes, devido principalmente ao uso intensivo de maneira indiscriminada e equivocada desses produtos

'Departamento de Medicina Veterinária Preventiva (DMVP), Centro de Ciências Rurais (CCR), Universidade Federal de Santa Maria (UFSM), Av. Roraima, 1000, Prédio 44, Sala 5131, Bairro Camobi, 97105-900, Santa Maria, RS, Brasil. E-mail: lasangioni@gmail.com. *Autor para correspondência.

"Departamento de Farmácia Industrial (DFI), Centro de Ciências da Saúde (CCS), UFSM, Santa Maria, RS, Brasil. 
(FURLONG etal., 2002; VARGAS et al., 2003). No Brasil, a resistência do $\boldsymbol{R}$. (B.) microplus aos acaricidas foi relatada frente aos diferentes grupos químicos (CAMPOS JÚNIOR \& OLIVEIRA, 2005; CAMILLO et al., 2009). Segundo FURLONG et al. (2004), em alguns estados brasileiros não existem mais produtos capazes de controlar eficientemente os carrapatos existentes.

Pesquisas buscando medidas alternativas de controle acaricida acentuaram-se a partir da década de 90, destacando-se os estudos da efetividade dos fitoterápicos. Esses produtos quando comparados aos produtos químicos são mais seguros, de custo reduzido, além de causarem menos danos ao ecossistema (AVANCINI, 1994; HEIMERDINGER et al., 2006). Agrega-se também a expansão da agricultura orgânica, considerando-se que essa estratégia de produção não permite o uso de pesticidas sintéticos.

Dentre as plantas com atividade terapêutica reconhecida popularmente, pode-se destacar a Glechon spathulata. O gênero Glechon, pertencente à tribo Mentheae, seção Glechonae, é um gênero exclusivamente sul-americano e distribui-se desde o sul do Brasil, compreendendo Minas Gerais, São Paulo, Paraná, Santa Catarina e Rio Grande do Sul, passando pelo Paraguai, Uruguai ao noroeste da Argentina (XIFREDA \& MALLO, 2004). No Rio Grande do Sul, existem 4 espécies do gênero: $\boldsymbol{G}$ spathulata Benth., $\boldsymbol{G}$ thymoides Spreng., G. marifolia Benth. e G. squarosa Benth. (DI PRIMIO, 1946). A análise fitoquímica revelou a presença de flavonóides, óleos voláteis, antocianinas, taninos condensados e, em menor quantidade, saponinas, triterpenos e taninos hidrolisáveis. Os taninos são compostos fenólicos de interesse econômico e farmacológico (KUNZ, 2007) e possuem atividade antiparasitária, demonstrada em endoparasitas e ainda não relatada em ectoparasitas (CAVALCANTE, et al., 2009).

Considerando-se a importância de pesquisas visando à descoberta de plantas com atividade acaricida, este trabalho teve como objetivo avaliar a ação in vitro de soluções contendo extrato bruto seco (EBS) e fração clorofórmica (FC) de $\boldsymbol{G}$. spathulata sobre teleóginas de $\boldsymbol{R}$. (B) microplus, com a finalidade de utilizá-la futuramente no controle deste carrapato em animais de produção.

\section{MATERIAL E MÉTODOS}

O material vegetal foi coletado no município de Santa Maria, Rio Grande do Sul, Brasil, sendo constituído por folhas de G. spathulata, conforme exsicata SMDB 10297, depositada no herbário do Departamento de Botânica da Universidade Federal de
Santa Maria (UFSM). No Laboratório de Farmacognosia da UFSM, esse material foi submetido à desidratação e estabilização em estufa a $40^{\circ} \mathrm{C}$, moagem em macromoinho de facas e estocagem em frasco âmbar e, posteriormente, foi macerado em etanol a $70 \%$. O solvente foi volatilizado em evaporador rotatório, obtendo-se o EBS. Parte deste foi ressuspendido em água e submetido ao fracionamento com solventes de polaridade crescente, obtendo-se a FC de $\boldsymbol{G}$ spathulata.

As teleóginas de $\boldsymbol{R}$. (B) microplus foram coletadas manualmente de bovinos mestiços de uma propriedade rural do município de São Pedro do Sul, Rio Grande do Sul, Brasil. Em seguida, elas foram acondicionadas em temperatura de aproximadamente $4^{\circ} \mathrm{C}$ e transportadas até o Laboratório de Doenças Parasitárias da UFSM, onde foram pesadas e selecionadas as teleóginas com comprimento entre 4,5 a $7,5 \mathrm{~mm}$. Os testes in vitro foram realizados em um intervalo máximo de 48 horas após a coleta.

A técnica de biocarrapaticidograma foi realizada conforme DRUMOND et al. (1973). Para tal, o EBS de G spathulata foi ressuspendido, parte em etanol a $70 \%$ e parte em água e a FC somente em água, ficando as soluções acertadas para a concentração final de $2 \%$. Como controle positivo, foi utilizada uma emulsão aquosa contendo Cipermetrina a 15\%, Clorpirifós a 25\% e Citronela a 1\% ( ${ }^{\circledR}$ Colosso-Ouro Fino) e, como controle negativo, foram usados água destilada e etanol a $70 \%$. Os testes utilizando extratos vegetais foram realizados em triplicatas e após calculou-se a média dos resultados.

Dessa forma, utilizaram-se seis tratamentos, sendo: T1- controle água destilada, T2- controle etanol a 70\%, T3- controle positivo (emulsão aquosa de Cipermetrina a $15 \%$, Clorpirifós a $25 \%$ e Citronela a $1 \%)$, T4- EBS $\boldsymbol{G}$ spathulata a $2 \%$ em água, T5- EBS $\boldsymbol{G}$ spathulata a $2 \%$ em etanol a $70 \%$; T6- FC de $\boldsymbol{G}$. spathulata em água destilada.

A análise estatística dos resultados obtidos foi realizada pelo teste Qui-quadrado (SAS, 2000), em nível de 5\% de significância $(\mathrm{P}<0,05)$.

\section{RESULTADOS}

Na triplicata de tratamentos utilizando EBS de $\boldsymbol{G}$ spathulata ressuspendido em etanol a 70\%, observou-se eclodibilidade média de $10 \%$ e eficácia média de $99 \%$, sendo, $\pm 0,67$ o erro padrão (EP). A triplicata de tratamentos utilizando EBS de $\boldsymbol{G}$. spathulata diluído em água apresentou eclodibilidade média de $43 \%$. Com relação à eficácia dessa solução, obteve-se resultado médio de $72 \%$, sendo $\pm 20,63 \mathrm{EP}$. A solução composta pela FC de $\boldsymbol{G}$ spathulata preparada 
em água não apresentou eficácia na triplicata de testes realizados, observando-se $90 \%$ de eclodibilidade. O controle positivo não obteve eclodibilidade, apresentando eficácia de $100 \%$. Os resultados de eficácia dos extratos vegetais apresentaram um nível de significância estatística inferior a 5\%, sendo $\mathrm{P}<0,0001$. A eficácia média de cada triplicata de tratamentos apresentou diferença estatística significativa entre si $(\mathrm{P}<0,0001)$, pelo teste Quiquadrado (Tabela 1). No estudo, foi verificado que o peso dos ovos nos grupos tratados (T2; T4 e T5) foi inferior ao grupo controle (água) (dados não apresentados).

\section{DISCUSSÃO}

Os tratamentos utilizando EBS de $\boldsymbol{G}$. spathulata ressuspendido em etanol a $70 \%$ demonstraram atividade acaricida in vitro, sendo considerado um carrapaticida eficaz. Observou-se que o controle etanol a 70\% não interferiu no peso da postura das teleóginas, mas causou diferença na eclodibilidade, quando comparado ao controle água destilada (Tabela 1). Esse resultado é semelhante ao encontrado por SILVA et al. (2008), os quais observaram que o tratamento com álcool reduziu a eclodibilidade, porém não interferiu na eficiência reprodutiva. Essa variável pode ser explicada por inúmeros fatores que alteram a eclodibilidade e viabilidade dos ovos no meio ambiente, tais como: umidade, temperatura, produção de guanina e quantidade de cera protetora produzida nos ovos, dentre outros (PEREIRA et al., 2008). CHAGAS et al. (2003) avaliaram a sensibilidade de larvas e fêmeas ingurgitadas de $\boldsymbol{R}$. (B) microplus a solventes e observaram que apenas o etanol $100 \%$

Tabela 1 - Eclodibilidade e eficácia média in vitro de diferentes tratamentos sobre teleóginas de $\boldsymbol{R}$. (Boophilus) microplus.

\begin{tabular}{lcl}
\hline Tratamento & $\begin{array}{c}\text { Eclodibilidade } \\
(\%)\end{array}$ & $\begin{array}{c}\text { Eficácia Médias } \\
(\%) \pm \mathrm{EP}\end{array}$ \\
\hline T1 & 95 & - \\
T2 & 50 & - \\
T3 & 0 & $100 \pm 0$ \\
T4 & 43 & $72^{\mathrm{b}} \pm 20,63$ \\
T5 & 10 & $99^{\mathrm{a}} \pm 0,67$ \\
T6 & 90 & $0^{\mathrm{c}} \pm 0,00$ \\
\hline
\end{tabular}

Letras distintas na coluna significam diferença pelo teste QuiQuadrado. T1- água destilada; T2- etanol a 70\%; T3- controle positivo (Cipermetrina a $15 \%$; Clorpirifós a $25 \%$ e Citronela a $1 \%$ ); T4- EBS Glechon spathulata a $2 \%$ em água; T5- EBS $\boldsymbol{G}$. spathulata a $2 \%$ em etanol a 70\%; T6- Fração clorofórmica de $\boldsymbol{G}$. spathulata a $2 \%$ em água. causou mortalidade. Segundo esses autores, o etanol é considerado de baixa toxicidade, porém, nesta pesquisa, constatou-se que ele interferiu na eclodibilidade dos ovos.

Os resultados de eficácia dos tratamentos contendo EBS de G. spathulata em água destilada indicaram ação acaricida in vitro (Tabela 1). Entretanto, a legislação pertinente para a comercialização de carrapaticidas no país preconiza o valor mínimo de $95 \%$ para que um tratamento seja considerado eficaz (BRASIL, 1990). Logo, na concentração de $2 \%$, este extrato, não foi considerado eficaz. COSTA JÚNIOR et al. (2002) obtiveram resultados superiores utilizando água destilada como solvente, analisando Derris urucu (timbó) em cinco diferentes concentrações $(1,0 ; 2,5$; 5,0; 7,5 e 10,0 $\mathrm{mg} \mathrm{ml}^{-1}$ ), evidenciando eficácia de 92,4 e $97,8 \%$ para as concentrações de 7,5 e $10,0 \mathrm{mg} \mathrm{ml}^{-1}$, respectivamente. CLEMENTE et al. (2007) testaram a eficácia in vitro do extrato bruto aquoso de Sapindus saponaria em quatro concentrações diferentes, não observando valores significativos para ação acaricida tanto em fêmeas ingurgitadas quanto em larvas.

Em estudo realizado por BORGES et al. (2003), sobre os efeitos da Melia azedarach (cinamomo) sobre larvas e teleóginas de $\boldsymbol{R}$. (B) microplus, foi observado que, com solventes de menor polaridade (hexano e clorofórmio), os extratos de $\boldsymbol{M}$. azedarach apresentaram melhor eficácia, discordando dos resultados constantes neste experimento, em que a FC obtida do fracionamento do EBS de $\boldsymbol{G}$ spathulata com a utilização do clorofórmio não apresentou atividade acaricida. Porém, foi constatada uma atividade acaricida eficaz frente ao etanol $70 \%$, isso possivelmente ocorreu devido a melhor solubilização do EBS, que foi previamente extraído utilizando solventes alcoólicos.

A emulsão aquosa de cipermetrina a $15 \%$, clorpirifós a $25 \%$ e citronela a $1 \%$ pode ser considerada um acaricida eficaz sobre os ácaros da respectiva propriedade na qual foram coletados, conforme legislação pertinente à comercialização de carrapaticidas (BRASIL, 1990). Corroborando CAMILLO et al. (2009) na realização de testes in vitro, utilizando essa associação, os quais observaram eficácia superior a $95 \%$ em $60,9 \%$ das amostras provenientes de propriedades do estado do Rio Grande do Sul, Brasil.

Em relação ao mecanismo de ação in vitro dos metabólitos presentes no EBS de $\boldsymbol{G}$. spathulata, observou-se que esses metabólitos interferiram nos aspectos reprodutivos dos ácaros, pois se constataram diferenças na eclodibilidade dos ovos dos grupos tratados, quando comparados aos respectivos dos 
grupos controle (Tabela 1). Resultados semelhantes foram descritos por BROGLIO-MICHELETTI et al. (2009) que, testando extratos de frutos de A. muricata (graviola) e de flores de Syzygium malaccensis (jambo), evidenciaram alterações na reprodução dos ácaros, além de mortalidade das teleóginas. BORGES et al. (2003) trabalharam com extrato bruto de frutos de $\boldsymbol{M}$. azedarach a $0,25 \%$, verificando elevada taxa de mortalidade de larvas e alta eficácia sobre fêmeas ingurgitadas, sendo que o extrato não matou fêmeas adultas, mas inibiu total ou parcialmente a produção de ovos e a embriogênese. BORGES et al. (2005) testaram esse mesmo extrato in vivo, verificando que não houve interferência nos aspectos reprodutivos dos carrapatos.

Estudos fitoquímicos de frutos de $\boldsymbol{M}$. azedarach evidenciaram a presença de taninos, compostos fenólicos (DANTAS et al., 2000) e saponinas (SAITO \& LUCHINI, 1998) e a ação acaricida dessa planta foi relatada por BORGES et al. (2005). Considerando-se que esses metabólitos secundários são comuns aos presentes em $\boldsymbol{G}$ spathulata (KUNZ, 2007), sugere-se que a ação acaricida dessa planta seja devido à atividade dos componentes vegetais citados anteriormente, ou até mesmo ao sinergismo entre eles. LAPA (1999) afirma que a síntese química das substâncias, ainda que orientada pelas características genéticas da planta, é controlada por fatores do ecossistema como iluminação, calor, constituição do solo, umidade.

Os resultados apresentados neste trabalho demonstraram que o EBS de G. spathulata apresentou atividade acaricida in vitro, sendo a eficácia das soluções parcialmente dependente do solvente utilizado na sua recuperação. A FC de $\boldsymbol{G}$. spathulata não demonstrou atividade carrapaticida in vitro. Entretanto, outros estudos deveriam incluir diferentes solventes e concentrações dos extratos para melhor avaliar a eficácia deste fitoterápico. Este estudo constitui o primeiro relato de ação acaricida do EBS de G. spathulata.

\section{REFERÊNCIAS}

AVANCINI, C.A.M. Sanidade Animal na Agroecologia Atitudes ecológicas de sanidade animal e plantas medicinais em medicina veterinária. Porto Alegre: Fundação Gaia, 1994. 46p.

BORGES, L.M.F. et al. In vitro efficacy of extracts of Melia azedarach against the tick Boophilus microplus. Medical and Veterinary Entomology, v.17, p.228-231, 2003. Disponível em: <http://onlinelibrary.wiley.com/doi/10.1046/ j.1365-2915.2003.00426.x/pdf>. Acesso em: 09 fev. 2011. doi: $10.1046 / \mathrm{j} .1365-2915.2003 .00426 . x$.
BORGES, L.M.F. et al. Ação do extrato hexânico de frutos maduros de Melia azedarach (meliaceae) sobre Boophilus microplus (acari: Ixodidae) em bezerros infestados artificialmente. Revista de Patologia Tropical, v.34, n.1, p.53-59, 2005.

BRASIL, Ministério da Agricultura. Portaria n. 90, de 04 dez. 1989. Normas para produção, controle e utilização de produtos antiparasitários. Diário Oficial da União, 22 jan. 1990. Seção 1, Coluna 2. Disponível em: <http://www.jusbrasil.com.br/ diarios/953617/dou-secao-1-22-01-1990>. Online. Acesso em: 12 ago. 2011.

BROGLIO-MICHELETTI, S.M.F. et al. Extratos de plantas no controle de Rhipicephalus (Boophilus) microplus (Canestrini, 1887) (Acari: Ixodidae) em laboratório. Revista Brasileira de Parasitologia Veterinária, v.18, n.4, p.4448, 2009. Disponível em: <http://www.cbpv.com.br/rbpv/ documentos/1842009/rbpv.01804008.pdf>. Acesso em: 09 fev. 2011. doi:10.4322/rbpv.01804008.

CAMILLO, G. et al. Eficiência in vitro de acaricidas sobre carrapatos de bovinos no Estado do Rio Grande do Sul, Brasil. Ciência Rural, v.39, p.490-495, 2009. Disponível em: <http://www.scielo.br/pdf/cr/v39n2/a82cr262.pdf >. Acesso em: 09 fev. 2011.

CAMPOS JUNIOR, D.A.; OLIVEIRA, P.R. Avaliação in vitro da eficiência de acaricidas sobre Boophilus microplus (Canestrini, 1887) (Acari: Ixodidae) de bovinos no município de Ilhéus, Bahia, Brasil. Ciência Rural, v.35, n.6, p.13861392, 2005. Disponível em: <http://www.scielo.br/pdf/cr/ v35n6/a25v35n6.pdf>. Acesso em: 09 fev. 2011.

CAVALCANTE, A.C.R. et al. Doenças parasitárias de caprinos e ovinos: epidemiologia e controle. Brasília-DF: Embrapa Informação Tecnológica, 2009. 603p.

CHAGAS, A.C.S. et al. Sensibilidade do carrapato Boophilus microplus a solventes. Ciência Rural, v.33, n.1, p.109-114, 2003. Disponível em: <http://www.scielo.br/pdf/cr/v33n1/ 14151.pdf>. Acesso em: 09 fev. 2011.

CLEMENTE, M.A et al. Avaliação do potencial de plantas medicinais no controle de Boophilus microplus (Acari: Ixodidae). Revista Brasileira de Biociências, v.5, n.2, p. 516-518, 2007. Disponível em: <http://www6.ufrgs.br/seerbio/ojs/index.php/rbb/ article/viewFile/460/404>. Acesso em: 09 fev. 2011.

COSTA JÚNIOR, L.M. et al. Eficiência "in vitro" de Rotenóides extraídos do Timbó (Derris urucu) em teleóginas do carrapato Boophilus microplus. In: CONGRESSO BRASILEIRO DE PARASITOLOGIA VETERINÁRIA, 12., 2002, Rio de Janeiro, RJ. Anais... Rio de Janeiro: CBBPV, 2002. 1CD ROOM.

DANTAS, D. A. et al. Estudo fitoquímico dos frutos de Melia azedarach L. (Cinamomo, Meliaceae). In: ENCONTRO DE PESQUISA E INICIAÇÃO CIENTÍFICA DA UNIDERP, 2., 2000, Campo Grande, MS. Anais... Campo Grande: UNIDERP, 2000. p.119-120.

DI PRIMIO, R.F. Flora do Rio Grande do Sul. Porto Alegre: Oficinas Gráficas da Imprensa Oficial, 1946. p.244-247.

DRUMOND, R.O. et al. Boophilus annulatus and Boophilus microplus: Laboratory tests of insecticides. Journal Economic Entomology, v.66, n.1, p.130-133, 1973. 
FARIAS, N.A.R. Situación de la resistência de la garrapata Boophilus microplus em la región sur de Rio Grande Del Sur, Brazil. In: SEMINÁRIO INTERNACIONAL DE PARASITOLOGIA ANIMAL, 4., 1999, Puerto Vallarta, México. Anais... Puerto Vallarta, Jalisco, 1999. p.25-30.

FRAGA, A.B. et al. Análise de fatores genéticos e ambientais que afetam a infestação de fêmeas bovinas da raça Caracu por carrapatos (Boophilus microplus). Revista Brasileira de Zootecnia, v.32, n.6, p.1578-1586, 2003. Disponível em: <http://www.scielo.br/pdf/rbz/v32n6s1/19676.pdf>. Acesso em: 09 fev. 2011.

FURLONG, J. et al. CL50 e CL90 dos extratos alcoólico e aquoso de nim indiano (Azadirachta indica) em larvas de Boophilus microplus. In: CONGRESSO BRASILEIRO DE PARASITOLOGIA VETERINÁRIA, 12., 2002, Rio de Janeiro, RJ. Anais... Rio de Janeiro: Universidade Federal Rural do Rio de Janeiro, 2002. v.12.

FURLONG, J. et al. Diagnóstico "in vitro" da sensibilidade do carrapato Boophilus microplus a acaricidas. In: CONGRESSO BRASILEIRO DE PARASITOLOGIA VETERINÁRIA, 13., 2004, Ouro Preto, MG. Anais... Ouro Preto: CBPV, 2004. p.305.

HEIMERDINGER, A. et al. Extrato alcoólico de capim-cidreira no controle do Boophilus microplus em bovinos. Revista Brasileira de Parasitologia Veterinária, v.15, n.1, p.3739, 2006. Disponível em: <http://cbpv.com.br/rbpv/documentos/ 1512006/c15137_39.pdf>. Acesso em: 09 fev. 2011.

KUNZ, V.T. Glechon spathulata BENTH.: estudo fitoquímico e biológico. 2007. 88f. Dissertação (Mestrado em Ciências
Farmacêuticas) - Universidade Federal de Santa Maria, Santa Maria, RS. Disponível em: <http://cascavel.cpd.ufsm.br/tede/tde_busca/ arquivo.php?codArquivo=2946>. Acesso em: 09 fev. 2011 .

LAPA, A.J. et al. Farmacologia e toxicologia de produtos naturais. In: SIMOES, C.M.O.; SCHENKEL, E.P. et al. Farmacognosia: da planta ao medicamento. Porto Alegre: UFRGS, 1999. p.181-196.

PEREIRA, M.C. Rhipicephalus (Boophilus) microplus: biologia, controle e resistência. São Paulo: Medvet. 2008. 192p.

SAITO, M.L.; LUCHINI, F. Substâncias obtidas de plantas e a procura por praguicidas eficientes e seguros ao meio ambiente. Jaguariúna: EMBRAPA/CNPMA, 1998. 46p. Disponível em: <http://www.agencia.cnptia.embrapa.br/Repositorio/ Saito_SubstanciasObtidasPlantasProcura_000fdrbfar702wx5eo0a2nd xylbfy537.pdf>. Acesso em: 09 fev. 2011.

SAS-INSTITUTE. SAS/STATO. User's guide: statistics, version 8.1.4. Carey, 2000. V.2. coref: 23.

VARGAS M.S. et al. Avaliação in vitro de uma cepa de campo de Boophilus microplus (Acari: Ixodidae) resistente à Amitraz. Ciência Rural, v.33, n.4, p.737-742, 2003. Disponível em: <http://www.scielo.br/pdf/cr/v33n4/16698.pdf>. Acesso em: 09 fev. 2011 .

XIFREDA, C.C.; MALLO, A.C. Las especies argentinas de Glechon (Lamiaceae, Mentheae). Darwiniana, v.42, p.333-346, 2004. 